\begin{tabular}{|r|l|}
\hline \multicolumn{2}{|c|}{ Statistica Sinica Preprint No: SS-2020-0405 } \\
\hline Title & $\begin{array}{l}\text { New Tests for High-Dimensional Linear Regression } \\
\text { Based on Random Projection }\end{array}$ \\
\hline Manuscript ID & SS-2020-0405 \\
\hline URL & http://www.stat.sinica.edu.tw/statistica/ \\
\hline DOI & $10.5705 /$ ss.202020.0405 \\
\hline Complete List of Authors & $\begin{array}{l}\text { Changyu Liu, } \\
\text { Xingqiu Zhao and } \\
\text { Jian Huang }\end{array}$ \\
\hline Corresponding Author & Xingqiu Zhao \\
\hline E-mail & xingqiu.zhao@polyu.edu.hk \\
\hline Notice: Accepted version subject to English editing.
\end{tabular}


Statistica Sinica

\title{
New Tests for High-Dimensional Linear Regression Based on Random Projection
}

\author{
Changyu Liu, Xingqiu Zhao \\ Department of Applied Mathematics, The Hong Kong Polytechnic University, Hong Kong, China \\ Jian Huang \\ Department of Statistics and Actuarial Science, University of Iowa, Iowa, U.S.A.
}

Abstract: We consider the problem of detecting the significance in high-dimensional linear models, allowing the dimension of the regression coefficient to be greater than the sample size. We propose novel test statistics for the hypothesis testing of testing the global significance of the linear model as well as the significance of part of the regression coefficients. The new tests are based on randomly projecting high-dimensional data into a space of low dimensions and then working with the classical F-test using the projected data. An appealing feature of the proposed tests is that they have a simple form and are computationally easy to implement. We derive the asymptotic local power functions of the proposed tests and compare with the existing methods for hypothesis testing in high-dimensional linear models. We also provide a sufficient condition under which our proposed tests have higher asymptotic relative efficiency. Through simulation studies, we evaluate the finite-sample performances of the proposed tests and demonstrate that it performs better than the existing tests in the models we considered. Applications to real high-dimensional gene expression data are also provided for illustration.

Key words and phrases: High-dimensional inference; Hypothesis testing; Linear model; Random projection; Relative efficiency. 


\section{Introduction}

High-dimensional data are now routinely encountered in many fields of scientific research. For example, in genomic studies, the dimension of many types of data such as gene expression and genetic marker data are typically far greater than the sample size. A common feature of high-dimensional data is that the data dimension $p$ can be greater than the sample size n. This phenomenon brings challenges to the classical statistical analysis even in many basic settings. For example, the Hotelling $T^{2}$ statistic for the two-sample testing problem is not well-defined when $p$ is larger than $n$, since the sample covariance matrix is no longer invertible in this setting. In high-dimensional linear regression models, existing methods for statistical inference about regression coefficients are no longer applicable. Therefore, it is important to develop new approaches for statistical inference in high-dimensional models.

Consider a linear regression model

$$
y_{i}=\alpha+\mathbf{x}_{i}^{\top} \boldsymbol{\beta}+\epsilon_{i}, \quad i=1, \ldots, n
$$

where $y_{i}$ is a response variable, $\mathbf{x}_{i}$ is a $p \times 1$ covariate vector, $\alpha$ is an intercept term, $\boldsymbol{\beta}$ is a $p \times 1$ vector of unknown coefficients, and $\epsilon_{i}$ is a random error term with mean zero and variance $\sigma^{2}$. We focus on the high-dimensional settings when $p$ can exceed the sample size $n$. We are interested in testing the hypothesis

$$
\mathbf{H}_{0}: \boldsymbol{\beta}=\mathbf{0} \quad \text { versus } \quad \mathbf{H}_{1}: \boldsymbol{\beta} \neq \mathbf{0}
$$


In low-dimensional settings, a basic test statistic for this problem is the F-test (Searle and Gruber, 2017). The idea behind this test is the least squares method which is based on projecting the vector of response variables onto the space generated by covariates. Under conditions $p<n$ and $y_{i} \mid \mathbf{x}_{i} \sim \mathcal{N}\left(\alpha+\mathbf{x}_{i}^{\top}, \sigma^{2}\right)$, the exact distribution of the F-test is known and has certain optimal properties since it can be considered as a likelihood ratio statistic. Without the normality assumption, Wang and Cui (2013) proposed a generalized F-test statistic and showed that it is asymptotically normal when $p / n \rightarrow \gamma$ with $\gamma \in(0,1)$. However, neither the F-test nor the generalized F-test is well-defined when $p \geq n$. Even when $p<$ $n$, Zhong and Chen (2011) showed that the F-test is adversely affected by the increasing dimension of covariates and has a poor performance. Recently, there has been much effort devoted to developing new test statistics for (1.2) that are applicable under the $p>n$ settings. Zhong and Chen (2011) proposed a test based on an U-statistic and extended it to accommodate factorial designs. This approach was further considered in Cui, Guo, and Zhong (2018) by implementing a new variance estimation method of Fan, Guo, and Hao (2012). Lan, Wang, and Tsai (2014) proposed a test for general random design, and Lan et al. (2016) focused on situation with highly correlated predictors. Based on the low dimensional projection (LDP) method, many statistical tests were proposed under sparsity condition. For example, statistical tests for single or low-dimensional components in highdimensional models were proposed in Zhang and Zhang (2014), Van de Geer et al. (2014), Javanmard and Montanari (2014), Javanmard and Montanari (2014) and Ning and Liu (2017). For global testing problems, Zhang and Cheng (2017) and Ma, Tony Cai, and Li (2020) constructed maximal-type statistics based on the LDP method. For linear hypotheses 
testing problems, Zhu and Bradic (2018) proposed a test applicable in nonsparse linear models, and Shi et al. (2019) constructed tests based on the constrained partial regularization method. However, these test statistics are relatively complicated in form and tend to be computationally expensive.

In this paper, we propose a new statistical test for hypothesis (1.2) in high-dimensional settings. Using the technique of random projection to reduce the data dimension, we construct F-statistics based on projected data which live in lower-dimensional space. The F-test based on projected data has a simple form and is easy to compute. Random projection has been applied to several high-dimensional statistical inference problems, including independence testing (Huang and Huo, 2017), two-sample testing (Lopes, Jacob, and Wainwright, 2011) and nonparametric testing (Liu, Shang, and Cheng, 2018). An important advantage of random projection based approach stems from its ability in dimension reduction while preserving the significant information in data simultaneously. The proposed test is shown to be applicable in a general situation under some mild conditions. The usage of random projection injects extra randomness to the test statistic, which requires further investigation of the relationship between the response and the projected data as well as the performance of the new hat matrix. Our analysis is inspired by the results that almost all low dimensional projection data is close to normal, according to Diaconis and Freedman (1984). Under the null hypothesis, it is shown that the proposed test statistic is asymptotically normal as $(n, p) \rightarrow \infty$. We also derive the asymptotic local power functions of the proposed tests. The results show that the asymptotic performance of the test statistics is similar to that in the setting when the data is normal, and demonstrate the benefit of the using random projection for reducing the 
dimension of the data. Finally, we extend the proposed random-projection-based test procedure for the global hypothesis (1.2) to the problem of testing partial regression coefficient and derive its asymptotic null distribution and local power function.

The rest of this paper is organized as follows. In Section 2, we propose our test statistic and give the intuition for its design. In Section 3, we establish the asymptotic null distribution of the proposed test statistic and derive its asymptotic local power function. We also derive the asymptotic relative efficiency of the proposed test in comparison with some recent tests. In Section 4, we extend the proposed test to the problem of testing partial regression coefficient and establish its asymptotic theoretical results. In Section 5.1, we conduct simulation studies to evaluate the finite-sample behavior of the proposed test in terms of type 1 error and power, and compare it with the competing test. We also illustrate its applications to high-dimensional gene expression data sets in 5.2 . Some conclusions are made in Section 6. The proofs of lemmas and theorems, and additional numerical studies are given in the supplementary materials.

\section{Test Statistic}

Let $\mathbf{x}_{i}=\left(x_{i 1}, \ldots, x_{i p}\right)^{\top}$ be the $i$ th row of the design matrix $\mathbf{X}=\left(\mathbf{x}_{1}, \ldots, \mathbf{x}_{n}\right)^{\top}$ and $\mathbf{y}=$ $\left(y_{1}, \ldots, y_{n}\right)^{\top}$. The linear model 1.1 can be written as

$$
\mathbf{y}=\alpha \mathbf{1}+\mathbf{X} \boldsymbol{\beta}+\boldsymbol{\epsilon}
$$

with the error vector $\boldsymbol{\epsilon}=\left(\epsilon_{1}, \ldots, \epsilon_{n}\right)^{\top}$ and $\mathbf{1}=(1, \ldots, 1)^{\top}$. 
To motivate the proposed test, we first recall the classical $F$-test of overall significance for regression in the $n>p$ settings. For simplicity, we consider the model without intercept

$$
\mathbf{y}=\mathbf{X} \boldsymbol{\beta}+\boldsymbol{\epsilon}
$$

We assume $\mathbf{X}$ is full column rank. Let $\mathbf{H}=\mathbf{X}\left(\mathbf{X}^{\top} \mathbf{X}\right)^{-1} \mathbf{X}^{\top}$ be the projection matrix (or hat matrix) for the regression. The $F$-statistic for testing $H_{0}: \boldsymbol{\beta}=0$ is

$$
F_{n}=\frac{\mathbf{y}^{\top} \mathbf{H y} / p}{\mathbf{y}^{\top}(\mathbf{I}-\mathbf{H}) \mathbf{y} /(n-p)}
$$

Under the normality assumption $\mathbf{y} \mid \mathbf{X} \sim \mathcal{N}\left(\mathbf{X} \boldsymbol{\beta}, \sigma^{2} \mathbf{I}\right), F_{n}$ has a noncentral $F$-distribution with degrees of freedom $(p, n-p)$. The $F$-test can be derived in different ways. For example, it can be derived based on the distribution of the least squares estimator of $\boldsymbol{\beta}$, and it can also be derived as a likelihood ratio test. Indeed, $F$-test is the most widely used methods for testing hypothesis about regression coefficients in linear models and enjoys certain optimality properties. In addition, it has a known finite-sample distribution and it is uniformly most powerful invariant (Lehmann, 1959). Clearly, the F-test in 2.3 is not applicable to highdimensional data with $n<p$.

To overcome this difficulty, we first project high-dimensional predictors onto a lowerdimensional space, and then apply the $F$-test to projected data. Specifically, for an integer $1 \leq k<\min \{n, p\}$, let $\mathbf{P}_{k} \in \mathbb{R}^{p \times k}$ denote a random projection matrix with random entries, drawn independently of the data. Define $\mathbf{u}_{k i}=\mathbf{P}_{k}^{\top} \mathbf{x}_{i}$. Let $\mathbf{U}_{k}=\left(\mathbf{u}_{k 1}, \ldots, \mathbf{u}_{k n}\right)^{\top}=\mathbf{X P}_{k}$. We 
consider a working model

$$
\mathbf{y}=\mathbf{U}_{k} \boldsymbol{\eta}+\boldsymbol{\epsilon} .
$$

We use this model to motivate the proposed test statistic. Of course, model (2.4) is generally different from model (2.2). However, for the purpose of constructing a valid test, it suffices that the null hypothesis $\mathbf{H}_{0}: \boldsymbol{\beta}=\mathbf{0}$ under model $(2.2)$ is equivalent to the null hypothesis $\mathbf{H}_{0}: \boldsymbol{\eta}=\mathbf{0}$ under $(2.4)$. To see this, we focus on a random projection $\mathbf{P}_{k}$ with i.i.d. $\mathcal{N}(0,1)$ entries. First, for $\boldsymbol{\eta}=\mathbf{0}$, model $(2.4)$ can be written as $\mathbf{y}=\boldsymbol{\epsilon}=\mathbf{X} \mathbf{0}+\boldsymbol{\epsilon}$. Therefore, $\mathbf{y}$ has the same distribution in model $(2.2)$ for $\boldsymbol{\beta}=\mathbf{0}$. Second, for $\boldsymbol{\eta} \neq \mathbf{0}, \mathbf{P}_{k} \boldsymbol{\eta} \neq \mathbf{0}$ holds with probability 1 , since $\mathbf{P}_{k} \boldsymbol{\eta}$ is distributed as $\mathcal{N}\left(\mathbf{0},\|\boldsymbol{\eta}\|_{2}^{2} \mathbf{I}\right)$. Consequently, $\boldsymbol{\beta}=\mathbf{0}$ in model (2.2) implies $\boldsymbol{\eta}=\mathbf{0}$ in model (2.4), otherwise, a contradiction will be led by $\mathbf{P}_{k} \boldsymbol{\eta} \neq \mathbf{0}$. Now suppose $\mathbf{U}_{k}$ is full column rank (this can be guaranteed if $k<n$ and $\mathbf{X}$ is full row rank). The projection matrix for $(2.4)$ is

$$
\mathbf{H}_{k}=\mathbf{U}_{k}\left(\mathbf{U}_{k}^{\top} \mathbf{U}_{k}\right)^{-1} \mathbf{U}_{k}^{\top}
$$

The $F$-statistic based on (2.4) is

$$
T_{n}=\frac{\mathbf{y}^{\top} \mathbf{H}_{k} \mathbf{y} / k}{\mathbf{y}^{\top}\left(\mathbf{I}-\mathbf{H}_{k}\right) \mathbf{y} /(n-k)}
$$

For the model with an intercept, $\mathbf{y}=\alpha \mathbf{1}+\mathbf{X} \boldsymbol{\beta}+\boldsymbol{\epsilon}$, we can simply center the design 
matrix and modify the test statistic as:

$$
T_{n}=\frac{\mathbf{y}^{\top} \mathbf{H}_{k} \mathbf{y} / k}{\mathbf{y}^{\top}\left(\mathbf{I}-\mathbf{P}_{1}-\mathbf{H}_{k}\right) \mathbf{y} /(n-k-1)},
$$

where $\mathbf{P}_{1}=\frac{1}{n} \mathbf{1} \mathbf{1}^{\top}$ and $\mathbf{H}_{k}=\mathbf{U}_{k}\left(\mathbf{U}_{k}^{\top} \mathbf{U}_{k}\right)^{-1} \mathbf{U}_{k}^{\top}$ is a new hat matrix with $\mathbf{U}_{k}=\left(\mathbf{I}-\mathbf{P}_{1}\right) \mathbf{X} \mathbf{P}_{k}$.

Note that the matrix $\mathbf{U}_{k}^{\top} \mathbf{U}_{k}$ is of full rank with probability 1 when $\mathbf{P}_{k}$ has i.i.d. $\mathcal{N}(0,1)$ entries, which ensures the new hat matrix is well-defined even when $p>n$ as shown in the proof of Theorem 3.1.

From the definition, the new test is based on a projection of the response vector $\mathbf{y}$ onto the space spanned by the columns of $\mathbf{U}_{k}$, which is a linear subspace of the space spanned by the columns of the centered $\mathbf{X}$.

A convenient way to construct $\mathbf{P}_{k}$ is to generate its entries as i.i.d. random variables from the standard normal distribution $N(0,1)$. Li, Hastie, and Church (2006) suggested that one can also generate other types of random projections $\mathbf{P}_{k}$, for example, sparse random projections, to achieve asymptotically the same performance as the normal random projection at a fast convergence rate. A sparse random projection consists of entries $p_{i j}$ that are i.i.d. from distributions satisfying

$$
P\left(p_{i j}=\sqrt{l}\right)=P\left(p_{i j}=-\sqrt{l}\right)=\frac{1}{2 l}, P\left(p_{i j}=0\right)=1-\frac{1}{l},
$$

where the choice of $l$ is recommended to be $\sqrt{p}$. Under this case, Li, Hastie, and Church (2006) showed that the entries of projected data converge to normal at a rate of $O\left(p^{-1 / 4}\right)$.

In our theoretical analysis, we will focus on random projections consisting of i.i.d. normal 
random entries. The results can be applied to some non-normal projections. We will use the above sparse random projection and evaluate the performance of non-normal projections in the simulation studies.

\section{Main Results}

This section consists of the statements of our main theoretical results and related discussions. Specifically, we derive the asymptotic normality and the asymptotic power function for the new random-projection-based test. We also conduct a comparison with one of the latest tests in terms of asymptotic relative efficiency.

\subsection{Asymptotic Normality}

Our first main result demonstrates the asymptotic normality of the standardized $T_{n}$ under the null hypothesis. We work under the following assumptions.

Assumption A1. $\boldsymbol{x}_{i}=\boldsymbol{\mu}+\boldsymbol{\Gamma} \boldsymbol{z}_{i}$, where $\boldsymbol{\Gamma}$ is a $p \times m$ matrix with $m \geq p$, $\boldsymbol{\mu}$ is a $p$-dimensional vector and $\boldsymbol{z}_{i}=\left(z_{i 1}, \ldots, z_{i m}\right)^{\top}$ is an m-variate random vector with $E\left(\boldsymbol{z}_{i}\right)=\boldsymbol{O}, \operatorname{Var}\left(\boldsymbol{z}_{i}\right)=\boldsymbol{I}_{m}$ and $\operatorname{Var}\left(\frac{z_{i}^{\top} z_{i}}{m}\right)=O\left(m^{-1}\right)$. For any nonnegative integers $q_{1}, \ldots, q_{m}$, with $\sum_{j=1}^{m} q_{j}=4$, the mixed moments $E\left(\Pi_{j=1}^{m} z_{i j}^{q_{j}}\right)$ are bounded, and equal to 0 when at least one of the $q_{j}$ is odd.

Assumption A2. $\mu_{4}=E\left(\epsilon_{1}^{4}\right)<\infty$.

Assumption A3. $p \gg n$ and there is a constant $\rho \in(0,1)$ such that $\frac{k}{n} \rightarrow \rho$.

As stated in Assumptions A1 and A3, we do not place any concrete relationships between $n$ and $p$, allowing the dimension $p$, mean vector $\boldsymbol{\mu}$ and covariance matrix $\boldsymbol{\Sigma}=\boldsymbol{\Gamma} \boldsymbol{\Gamma}^{\top}$ to 


\subsection{Asymptotic Normality}

implicitly vary as $n$ goes to infinity. This makes our test accommodate extremely highdimensional problems. Taking a closer look at Assumption A1, we find it resembles a factor model structure which has a linear relationship between $\mathbf{x}_{i}$ and $\mathbf{z}_{i}$. It can be proved that the following two kinds of assumptions are both included in Assumption A1.

D1 (Pseudo-independence assumption.) Suppose the $\mathrm{p}$-variate random vector $\mathbf{x}_{i}$ follows the general multivariate model: $\mathbf{x}_{i}=\boldsymbol{\mu}+\boldsymbol{\Gamma} \mathbf{z}_{i}$, where $\boldsymbol{\mu}$ is a $\mathrm{p}$-dimensional real vector, $\boldsymbol{\Gamma}$ is a $p \times m$ matrix, and $\mathbf{z}_{i}=\left(z_{i 1}, \ldots, z_{i m}\right)^{\top}$ is an $\mathrm{m}$-variate random vector with $E\left(\mathbf{z}_{i}\right)=\mathbf{0}$ and $\operatorname{Var}\left(\mathbf{z}_{i}\right)=\mathbf{I}_{m}$. Furthermore, each $z_{i j}$ satisfies $E\left(z_{i j}^{4}\right)=3+\Delta<\infty$ for some constant $\Delta$, and $E\left(z_{i j_{1}}^{l_{1}} \cdots z_{i j_{d}}^{l_{d}}\right)=E\left(z_{i j_{1}}^{l_{1}}\right) \cdots E\left(z_{i j_{d}}^{l_{d}}\right)$ for any $\sum_{v=1}^{d} l_{v} \leq 4$ and $j_{1} \neq \cdots \neq j_{d}$, where $\mathrm{d}$ is a positive integer. Integers $m$ and $p$ satisfy $m \geq p$.

D2 (Elliptical distribution assumption.) Suppose the p-variate random vector $\mathbf{x}_{i}$ satisfies the stochastic representation: $\mathbf{x}_{i}=\boldsymbol{\mu}+\boldsymbol{\Gamma} r_{i} \mathbf{u}_{i}$, where $\boldsymbol{\mu}$ is a p-dimensional real vector, $\boldsymbol{\Gamma}$ is a $p \times p$ matrix, $\mathbf{u}_{i}$ is a random vector uniformly distributed on the unit sphere in $\mathbb{R}^{p}$ and $r_{i}$ is a nonnegative random variable independent of $\mathbf{u}_{i}$ satisfying $E\left(r_{i}^{2}\right)=p$ and $\operatorname{Var}\left(r_{i}^{2}\right)=O(p)$.

The pseudo-independence assumption and its similar versions were used in Bai and Saranadasa 『 (1996), Zhong and Chen (2011), and Cui, Guo, and Zhong (2018). Such assumptions are similar to Assumption A1, but imposing stricter conditions on each element of $\mathbf{z}_{i}$. This is because $\mathbf{z}_{i}$ in D1 satisfies $\operatorname{Var}\left(\frac{\mathbf{z}_{i}^{\top} \mathbf{z}_{i}}{m}\right)=\frac{2+\Delta}{m}$. In the multivariate statistical analysis, elliptical distribution is often assumed to facilitate study. It includes a flexible family of distributions, including multivariate normal distribution, multivariate $t$-distribution and multivariate lo- 
gistic distribution. Let $\mathbf{z}_{i}=r_{i} \mathbf{u}_{i}$ and $m=p$, D2 and Assumption A1 enjoy a similar form. Furthermore, Lemma 3.1, together with Lemma A.1 in the supplementary material, indicates that the distributions satisfying $\mathrm{D} 2$ are included in Assumption $\mathrm{A} 1$.

Since $T_{n}$ is invariant to the location shift of $\mathbf{y}$ and $\mathbf{X}$, we assume that $\alpha=0$ and $\boldsymbol{\mu}=\mathbf{0}$ in the rest of the paper.

Lemma 3.1. Suppose $\boldsymbol{u}_{1}$ is a random vector uniformly distributed on the unit sphere in $\mathbb{R}^{p}$ and $r_{1}$ is a nonnegative random variable independent of $\boldsymbol{u}_{1}$ satisfying $E\left(r_{1}^{2}\right)=p$ and $\operatorname{Var}\left(r_{1}^{2}\right)=O(p)$. Let $\boldsymbol{z}_{1}=r_{1} \boldsymbol{u}_{1}$. Then

$$
E\left(\boldsymbol{z}_{1}\right)=\boldsymbol{O}, \operatorname{Var}\left(\boldsymbol{z}_{1}\right)=\boldsymbol{I}_{p}, \operatorname{Var}\left(\frac{\boldsymbol{z}_{1}^{\top} \boldsymbol{z}_{1}}{p}\right)=O\left(p^{-1}\right) .
$$

Therefore, our assumption for the distribution of $\mathbf{x}_{i}$ is relatively flexible. For example, there is no specific condition on the covariance matrix $\boldsymbol{\Sigma}$. For the error term, we only assume that $\epsilon_{i}$ is generated from a distribution having a finite fourth moment. The projection dimension $k$ is assumed to be asymptotically proportional to $n$ with a coefficient $\rho \in(0,1)$. The choice of $\rho$ will be discussed in the subsequent Section 3.3 .

Clearly, to derive the asymptotic distribution of $T_{n}$, we need to study the properties of the hat matrix $\mathbf{H}_{k}$. Since $\mathbf{H}_{k}=\mathbf{U}_{k}\left(\mathbf{U}_{k}^{\top} \mathbf{U}_{k}\right)^{-1} \mathbf{U}_{k}^{\top}$, the properties of $\mathbf{H}_{k}$ can be established when $\mathbf{U}_{k}$ is generated from Gaussian variables. Diaconis and Freedman (1984) showed that the empirical distribution of randomly projected data tends to be approximately Gaussian. Inspired by this result, we will show in Lemmas A.7 and A.8 in the supplementary material that $\mathbf{U}_{k}$ is asymptotically close to Gaussian, which demonstrates the advantage of the random 
projection method. We state the asymptotic distribution of the standardized $T_{n}$ under the null hypothesis.

Theorem 3.1. Suppose the random projection matrix $\boldsymbol{P}_{k}$ consists of i.i.d. standard normal random variables. Under Assumptions A1-A3 and $\boldsymbol{H}_{0}$, as $n \rightarrow \infty$, we have

$$
\frac{T_{n}-1}{\sqrt{2 / n \rho(1-\rho)}} \stackrel{\mathcal{D}}{\longrightarrow} \mathcal{N}(0,1) .
$$

This asymptotic normality result justifies the following test procedure. Given an $\alpha$-level of significance, the proposed test rejects $\mathbf{H}_{0}$ if

$$
\frac{T_{n}-1}{\sqrt{2 / n \rho(1-\rho)}}>z_{\alpha}
$$

where $z_{\alpha}$ is the upper $\alpha$-quantile of $\mathcal{N}(0,1)$.

\subsection{Asymptotic Power Function}

We now investigate the asymptotic power function of the proposed test. Additional assumptions are needed to facilitate our analysis.

Assumption A4. $\boldsymbol{\beta}^{\top} \boldsymbol{\Sigma} \boldsymbol{\beta}=o(1)$.

Assumption A4 is known as a local alternative, which is commonly used in studying the asymptotic properties of a statistical test. Detailed discussions can be found in van der Vaart (1998, Section 14.1).

In the classical F-test in 2.3 , the hat matrix $\mathbf{H}$ enjoys the properties $\mathbf{X}^{\top} \mathbf{H}=\mathbf{X}^{\top}$ and 
$\mathbf{H X}=\mathbf{X}$. Hence,

$$
\mathbf{y}^{\top} \mathbf{H y}=\boldsymbol{\beta}^{\top} \mathbf{X}^{\top} \mathbf{X} \boldsymbol{\beta}+\mathbf{2} \boldsymbol{\beta}^{\top} \mathbf{X}^{\top} \boldsymbol{\epsilon}+\boldsymbol{\epsilon}^{\top} \mathbf{H} \boldsymbol{\epsilon}
$$

where $\boldsymbol{\epsilon}^{\top} \mathbf{H} \boldsymbol{\epsilon}$ does not involve the parameter value. It indicates that the power of the F-test relies on $\boldsymbol{\beta}^{\top} \mathbf{X}^{\top} \mathbf{X} \boldsymbol{\beta}$ and $\boldsymbol{\beta}^{\top} \mathbf{X}^{\top} \boldsymbol{\epsilon}$. Thus, we can use the properties of $\mathbf{H}$ in the power analysis of the F-test without the need to consider the inverse of $\mathbf{X}^{\top} \mathbf{X}$.

However, the properties of $\mathbf{H}$ do not hold for the hat matrix $\mathbf{H}_{k}$. Fortunately, we can get around this problem based on the properties of random projection. Specifically, the fact that randomly projected variable is asymptotically normal yields a new representation for the model 2.1 by $\mathbf{y}=\mathbf{X} \mathbf{P}_{k} \boldsymbol{\xi}+\mathbf{e}$, where $\boldsymbol{\xi}=\left(\mathbf{P}_{k}^{\top} \boldsymbol{\Sigma} \mathbf{P}_{k}\right)^{-1} \mathbf{P}_{k}^{\top} \boldsymbol{\Sigma} \boldsymbol{\beta}$ and $\mathbf{e}=\mathbf{y}-\mathbf{X} \mathbf{P}_{k} \boldsymbol{\xi}$. Note that $\alpha$ is assumed to be zero here. It can be shown that the new error term e is asymptotically conditional independent of $\mathbf{X P} \mathbf{P}_{k}$, making the conventional analysis for the F-test applicable here. To rigorously show this, additional requirement for $\mathbf{z}_{i}$ is needed as follows.

Assumption A5. The m-variate random vector $\boldsymbol{z}_{i}=\left(z_{i 1}, \ldots, z_{i m}\right)^{\top}$ has a Lebesgue density $f_{\boldsymbol{z}}$ and satisfy $E\left(\boldsymbol{z}_{i}\right)=\boldsymbol{O}$ and $\operatorname{Var}\left(\boldsymbol{z}_{i}\right)=\boldsymbol{I}_{m}$. For $j=1, \ldots, m$, the components $z_{i j}$ are assumed to be independent, satisfy $E\left(z_{i j}^{20}\right) \leq C$ for a constant $C$, and have the marginal density bounded by a constant $D \geq 1$

Define $\delta_{k}^{2}=\sigma^{2}+\boldsymbol{\beta}^{\top} \boldsymbol{\Sigma} \boldsymbol{\beta}-\boldsymbol{\xi}^{\top} \mathbf{P}_{k}^{\top} \boldsymbol{\Sigma} \mathbf{P}_{k} \boldsymbol{\xi}$ to be the variance of the new error. We derive the asymptotic power function of the proposed test.

Theorem 3.2. Suppose Assumptions A1 A5 hold. Let $\Psi_{n}^{R P}\left(\boldsymbol{\beta} ; \boldsymbol{P}_{k}\right)$ denote the power func- 
tion of the proposed random-projection-based test $T_{n}$. Then

$$
\Psi_{n}^{R P}\left(\boldsymbol{\beta} ; \boldsymbol{P}_{k}\right)-\Phi\left(-z_{\alpha}+\sqrt{\frac{n(1-\rho)}{2 \rho}} \frac{\boldsymbol{\xi}^{\top} \boldsymbol{P}_{k}^{\top} \boldsymbol{\Sigma} \boldsymbol{P}_{k} \boldsymbol{\xi}}{\delta_{k}^{2}}\right) \rightarrow 0
$$

where $\Phi(\cdot)$ is the cumulative distribution function of the standard normal distribution, and $z_{\alpha}$ is the upper $\alpha$-quantile of $\Phi$.

It is remarked that there is no extra assumption made for $\boldsymbol{\Sigma}$, showing that the power property of the proposed test holds over a wide range of alternatives. The asymptotic power function relies on $\mathbf{P}_{k}$ and is a increasing function of the product $\boldsymbol{\xi}^{\top} \mathbf{P}_{k}^{\top} \boldsymbol{\Sigma} \mathbf{P}_{k} \boldsymbol{\xi}$. It is found that the product is upper bounded by $\boldsymbol{\beta}^{\top} \boldsymbol{\Sigma} \boldsymbol{\beta}$, which can be reached when the vector $\boldsymbol{\Gamma}^{\top} \boldsymbol{\beta}$ is in the space generated by $\boldsymbol{\Gamma}^{\top} \mathbf{P}_{k}$. To make the bound achieved asymptotically, we give a sufficient condition.

Assumption A6. (Tail eigenvalue condition.) There exists an integer s and a real number $\gamma>0$ such that $s<k$ and $\|\boldsymbol{\beta}\|_{2}^{2} \sum_{i=s+1}^{p} d_{i}=o\left(p n^{-0.5-\gamma}\right)$, where $d_{i}$ are the eigenvalues of $\boldsymbol{\Sigma}$ satisfying $d_{1} \geq d_{2} \geq \cdots \geq d_{p} \geq 0$.

We call Assumption A6 a tail eigenvalue condition, as it requires the product of $\|\boldsymbol{\beta}\|_{2}^{2}$ and the sum of tail eigenvalues of $\Sigma$ to be of order less than $p / \sqrt{n}$.

Lemma 3.2. Let $\boldsymbol{P}_{k} \in \mathbb{R}^{p \times k}$ consist of i.i.d. $\mathcal{N}(0,1)$ entries. Assume that Assumption A6 holds. Then we have

$$
\sqrt{n}\left\|\boldsymbol{\Gamma}^{\top} \boldsymbol{\beta}-\boldsymbol{\Gamma}^{\top} \boldsymbol{P}_{k} \boldsymbol{\eta}\right\|_{2}^{2}=o(1)
$$

for some $\boldsymbol{\eta} \in \mathbb{R}^{k}$ with probability tending to one. 
This lemma indicates that we can approximate $\boldsymbol{\Gamma}^{\top} \boldsymbol{\beta}$ by $\boldsymbol{\Gamma}^{\top} \mathbf{P}_{k} \boldsymbol{\eta}$ with negligible approximation error. In this case, we denote the asymptotic power function as $\Psi_{n}^{R P}(\boldsymbol{\beta})$ since it is not related to $\mathbf{P}_{k}$. A formal result is given in the following corollary.

Corollary 3.1. Suppose Assumptions A1 A6 hold. Then

$$
\Psi_{n}^{R P}(\boldsymbol{\beta})-\Phi\left(-z_{\alpha}+\sqrt{\frac{n(1-\rho)}{2 \rho}} \frac{\boldsymbol{\beta}^{\top} \boldsymbol{\Sigma} \boldsymbol{\beta}}{\sigma^{2}}\right) \rightarrow 0
$$

where $\Phi(\cdot)$ is the cumulative distribution function of the standard normal distribution, and $z_{\alpha}$ is the upper $\alpha$-quantile of $\Phi$.

\subsection{Choice of $\rho$}

The proposed test can be applied with any dimension of the projected space $k$ that satisfy Assumption A3. However, the power of the test will depend on $\rho$. In this subsection, we will give detailed discussion for the choice of $\rho$.

From Theorem 3.2, the asymptotic local power function satisfies

$$
\Psi_{n}^{R P}\left(\boldsymbol{\beta} ; \mathbf{P}_{k}\right)=\Phi\left(-z_{\alpha}+\sqrt{\frac{n(1-\rho)}{2 \rho}} \frac{\boldsymbol{\xi}^{\top} \mathbf{P}_{k}^{\top} \boldsymbol{\Sigma} \mathbf{P}_{k} \boldsymbol{\xi}}{\delta_{k}^{2}}\right)+o(1)
$$

Let $\Delta_{k}^{2}=\boldsymbol{\xi}^{\top} \mathbf{P}_{k}^{\top} \boldsymbol{\Sigma} \mathbf{P}_{k} \boldsymbol{\xi} . \Delta_{k}^{2}$ can be derived by projecting the vector $\boldsymbol{\Gamma}^{\top} \boldsymbol{\beta}$ onto the space generated by $\boldsymbol{\Gamma}^{\top} \mathbf{P}_{k}$. Intuitively, larger $\rho$ would lead to larger $\Delta_{k}^{2}$, since the dimension of projection space $k=\rho n$ becomes larger. However, with the increase of $\rho$, the function $\sqrt{(1-\rho) / \rho}$ would become smaller. This indicates that choice of $\rho$ is a compromise between these two values. 
First, we consider a situation, where the condition given in Corollary 3.1 is satisfied. In this case, $\Delta_{k}^{2}$ becomes a deterministic value, even with randomly generated projection matrix $\mathbf{P}_{k}$. The asymptotic local power function is a decreasing function of $\rho$, confirmed in the simulation studies. And $\rho$ can be arbitrarily small as long as the tail eigenvalue condition is satisfied.

Then, we consider the other situation, where $\boldsymbol{\Sigma}=\mathbf{I}$. In this case, the eigenvalues of $\boldsymbol{\Sigma}$ are equally significant with $\Delta_{k}^{2}=\boldsymbol{\beta}^{\top} \mathbf{P}_{k}\left(\mathbf{P}_{k}^{\top} \mathbf{P}_{k}\right)^{-1} \mathbf{P}_{k}^{\top} \boldsymbol{\beta}$. Suppose that direction of $\boldsymbol{\beta}$ is uniformly generated on the unit sphere. From Proposition 1 in Lopes, Jacob, and Wainwright (2011), quantity $\Delta_{k}^{2}$ satisfies

$$
P\left(\frac{\Delta_{k}^{2}}{\|\boldsymbol{\beta}\|_{2}^{2}} \geq \frac{c k}{p}\right) \rightarrow 1 \text { and } P\left(\frac{\Delta_{k}^{2}}{\|\boldsymbol{\beta}\|_{2}^{2}} \leq \frac{C k}{p}\right) \rightarrow 1
$$

for some constants $c$ and $C$. This indicates $\Delta_{k}^{2}$ scales linearly in k up to random fluctuations. Combining this result with (3.1), the influence of $\rho$ on the testing power is mainly achieved based on the function $g(\rho)=\sqrt{\frac{1-\rho}{\rho}} \cdot \rho$, which is maximized when $\rho=0.5$. Therefore, choice of $k=[0.5 n]$ may be asymptotically optimal in a general sense.

For most applications, where no prior information of $\boldsymbol{\Sigma}$ might be available, the above discussion suggests that $\rho$ around 0.5 would be an applicable choice, since the setting above can make the test performance reasonable, even in extreme cases. For some situations, where estimation methods of $\boldsymbol{\Sigma}$ or related function of $\boldsymbol{\Sigma}$ are available, $\rho$ could be selected based on the estimators. For example, the ratio $\operatorname{tr}(\boldsymbol{\Sigma})^{2} / \operatorname{tr}\left(\boldsymbol{\Sigma}^{2}\right)$, which lies between 1 and p, can be viewed as measuring the decay rate of the spectrum of $\boldsymbol{\Sigma}$ (Lopes, Jacob, and Wainwright, 
2011). And the tail eigenvalue condition could be satisfied when $\operatorname{tr}(\boldsymbol{\Sigma})^{2} / \operatorname{tr}\left(\boldsymbol{\Sigma}^{2}\right) \ll p$. Consequently, we could determine $\rho$ according to estimation of the ratio, which is available based on the estimators of $\operatorname{tr}(\boldsymbol{\Sigma})$ and $\operatorname{tr}\left(\boldsymbol{\Sigma}^{2}\right)$ proposed in Chen, Zhang, and Zhong (2010).

\subsection{Asymptotic Relative Efficiency (ARE)}

The asymptotic power function of the proposed random-projection-based test in Corollary 3.1 has the same form as the F-test, which was studied in Zhong and Chen (2011). However, our test accommodates high-dimensional settings and has milder assumptions on $\mathbf{X}$ and $\boldsymbol{\epsilon}$. As it is well-known that the $F$-test has good performance in low dimensions, the new test, as an extension of the F-test to high dimensions, is expected to perform well under certain conditions. To confirm this, we compare the performance of our test with the test proposed by Cui, Guo, and Zhong (2018), which is one of the latest tests designed for the testing problem (1.2) and is demonstrated to have superior performance over the existing tests for the problem considered. We denote this competing test by RCV test and show our test outperforms it in some situations. In this subsection, we suppose Assumption A6 holds.

With a slight abuse of notation, we also denote the asymptotic power function of our random-projection-based $(\mathrm{RP})$ test as

$$
\Psi_{n}^{R P}(\boldsymbol{\beta})=\Phi\left(-z_{\alpha}+\sqrt{\frac{n(1-\rho)}{2 \rho}} \frac{\boldsymbol{\beta}^{\top} \boldsymbol{\Sigma} \boldsymbol{\beta}}{\sigma^{2}}\right) .
$$

The asymptotic power function of the RCV test proposed by Cui, Guo, and Zhong 
(2018) is given by

$$
\Psi_{n}^{R C V}(\boldsymbol{\beta})=\Phi\left(-z_{\alpha}+\frac{n \boldsymbol{\beta}^{\top} \boldsymbol{\Sigma}^{2} \boldsymbol{\beta}}{\sigma^{2} \sqrt{2 \operatorname{tr}\left(\boldsymbol{\Sigma}^{2}\right)}}\right) .
$$

Since the term added to $-z_{\alpha}$ inside the $\Phi(\cdot)$ function is what controls power, the ratio of such terms can be defined as the asymptotic relative efficiency (ARE). For comparison, we define the ARE of our test to the RCV test as

$$
A R E\left(\Psi_{n}^{R P}, \Psi_{n}^{R C V}\right)=\left(\sqrt{\frac{n(1-\rho)}{\rho}} \boldsymbol{\beta}^{\top} \boldsymbol{\Sigma} \boldsymbol{\beta} / \frac{n \boldsymbol{\beta}^{\top} \boldsymbol{\Sigma}^{2} \boldsymbol{\beta}}{\sqrt{\operatorname{tr}\left(\boldsymbol{\Sigma}^{2}\right)}}\right)^{2} .
$$

Whenever the ARE is larger than 1, the proposed test is asymptotically powerful than the competing test. Therefore, we search for sufficient conditions under which the ARE is greater than 1 .

Write $\boldsymbol{\beta}$ as $\|\boldsymbol{\beta}\|_{2} \boldsymbol{\delta}$, where $\boldsymbol{\delta}=\frac{\boldsymbol{\beta}}{\|\boldsymbol{\beta}\|_{2}}$ is the direction of $\boldsymbol{\beta}$. Under Assumptions A4 and A6. we further require the sum of tail eigenvalues satisfying $\sum_{i=s+1}^{p} d_{i} / \boldsymbol{\delta}^{\top} \boldsymbol{\Sigma} \boldsymbol{\delta}=O\left(p n^{-0.5-\gamma}\right)$, where $\gamma$ is a small constant greater than zero. By Jensen's inequality, we have

$$
A R E\left(\Psi_{n}^{R P} ; \Psi_{n}^{R C V}\right) \geq \frac{1-\rho}{\rho} \frac{\sum_{i=s+1}^{p} d_{i}^{2}}{n}\left(\frac{\boldsymbol{\delta}^{\top} \boldsymbol{\Sigma} \boldsymbol{\delta}}{\boldsymbol{\delta}^{\top} \boldsymbol{\Sigma}^{2} \boldsymbol{\delta}}\right)^{2} \geq \frac{1-\rho}{\rho} \frac{\left(\boldsymbol{\delta}^{\top} \boldsymbol{\Sigma} \boldsymbol{\delta}\right)^{4}}{\left(\boldsymbol{\delta}^{\top} \boldsymbol{\Sigma}^{2} \boldsymbol{\delta}\right)^{2}} O\left(p n^{-2-2 \gamma}\right) .
$$

Clearly, if $\frac{\left(\boldsymbol{\delta}^{\top} \boldsymbol{\Sigma}^{2} \boldsymbol{\delta}\right)^{2}}{\left(\boldsymbol{\delta}^{\top} \boldsymbol{\Sigma} \boldsymbol{\delta}\right)^{4}}=o\left(p n^{-2-2 \gamma}\right)$, the right side of the inequality goes to infinity as $n$ goes to $\infty$, which sufficiently demonstrates that the proposed test is more powerful than the RCV test. In addition, this inequality shows that $\rho$ is preferred to be the smallest value such that the tail eigenvalue condition holds.

We give two examples to illustrate situations where $\frac{\left(\boldsymbol{\delta}^{\top} \boldsymbol{\Sigma}^{2} \boldsymbol{\delta}\right)^{2}}{\left(\boldsymbol{\delta}^{\top} \boldsymbol{\Sigma}\right)^{4}}=o\left(p n^{-2-2 \gamma}\right)$ is satisfied. 
Example 3.1. Suppose $\boldsymbol{\beta}$ is an eigenvector of $\boldsymbol{\Sigma}$, then $\frac{\left(\boldsymbol{\delta}^{\top} \boldsymbol{\Sigma}^{2} \boldsymbol{\delta}\right)^{2}}{\left(\boldsymbol{\delta}^{\top} \boldsymbol{\Sigma} \boldsymbol{\delta}\right)^{4}}=1$. Given that $n=$ $o\left(p^{1 /(2+2 \gamma)}\right)$ for a constant $\gamma>0$, which frequently happens when $p \gg n$, we have $\frac{\left(\boldsymbol{\delta}^{\top} \boldsymbol{\Sigma}^{2} \boldsymbol{\delta}\right)^{2}}{\left(\boldsymbol{\delta}^{\top} \boldsymbol{\Sigma} \boldsymbol{\delta}\right)^{4}}=$ $o\left(p n^{-2-2 \gamma}\right)$.

Example 3.2. Suppose the covariance matrix $\mathbf{\Sigma}$ has the spectral decomposition

$$
\boldsymbol{\Sigma}=\boldsymbol{O} \boldsymbol{\Lambda} \boldsymbol{O}^{\top}=\boldsymbol{O} \operatorname{diag}\left(d_{1}, \ldots, d_{p}\right) \boldsymbol{O}^{\top}
$$

where $\boldsymbol{O}$ is an orthogonal matrix with ith column denoted by $\boldsymbol{O}_{i}$, and $d_{i}$ are the eigenvalues of $\boldsymbol{\Sigma}$ satisfying $0 \leq d_{1} \leq d_{2} \leq \cdots \leq d_{p}$. We assume there exist integers $1 \leq s_{1} \leq s_{2} \leq p$ and constants $r_{1} \leq r_{2}$ such that, for $i=s_{1}, \ldots, s_{2}$, the order of $d_{i}$ is between $n^{r_{1}}$ and $n^{r_{2}}$ in the sense that $1 / d_{i}=O\left(n^{-r_{1}}\right)$ and $d_{i}=O\left(n^{r_{2}}\right)$. Consider $\boldsymbol{\beta} \in \operatorname{Span}\left\{\boldsymbol{O}_{s_{1}}, \ldots, \boldsymbol{O}_{s_{2}}\right\}$. Then we get

$$
\frac{\left(\boldsymbol{\delta}^{\top} \boldsymbol{\Sigma}^{2} \boldsymbol{\delta}\right)^{2}}{\left(\boldsymbol{\delta}^{\top} \boldsymbol{\Sigma} \boldsymbol{\delta}\right)^{4}} \leq O\left(n^{4\left(r_{2}-r_{1}\right)}\right)
$$

When $n$ and $p$ satisfy $n=o\left(p^{1 / 2\left(1+\gamma+2 r_{2}-2 r_{1}\right)}\right)$ for a constant $\gamma>0$, we have $\frac{\left(\boldsymbol{\delta}^{\top} \boldsymbol{\Sigma}^{2} \boldsymbol{\delta}\right)^{2}}{\left(\boldsymbol{\delta}^{\top} \boldsymbol{\Sigma} \boldsymbol{\delta}\right)^{4}}=$ $o\left(p n^{-2-2 \gamma}\right)$, and thus our test outperforms the RCV test in these situations.

\section{Testing Partial Regression Coefficients}

In Section 3, we proposed a random-projection-based test for the hypothesis testing (1.2). In many studies, we are also interested in investigating the significance of part of the covariates. In this section, we generalize the test in Section 3 to the hypothesis testing of partial linear regression coefficient and derive its asymptotic results. 
Consider a linear regression model

$$
y_{i}=\alpha+\mathbf{x}_{1 i}^{\top} \boldsymbol{\beta}_{1}+\mathbf{x}_{2 i}^{\top} \boldsymbol{\beta}_{2}+\epsilon_{i}, \quad i=1, \ldots, n,
$$

where $\alpha$ is an intercept term, $\mathbf{x}_{1 i}$ is a $p_{1}$-dimensional covariate and $\mathbf{x}_{2 i}$ is a $p_{2}$-dimensional covariate, $\boldsymbol{\beta}_{1}$ and $\boldsymbol{\beta}_{2}$ are vectors of unknown regression coefficients correspondingly, and $\epsilon_{i}$ is a random variable with mean zero and variance $\sigma^{2}$. We are interested in testing the hypotheses

$$
\mathbf{H}_{\text {part }, 0}: \boldsymbol{\beta}_{2}=\mathbf{0} \quad \text { versus } \quad \mathbf{H}_{\text {part }, 1}: \boldsymbol{\beta}_{2} \neq \mathbf{0}
$$

Let $\mathbf{y}=\left(y_{1}, \ldots, y_{n}\right)^{\top}$ and $\mathbf{x}_{1 i}=\left(x_{i 1}^{1}, \ldots, x_{i p_{1}}^{1}\right)^{\top}$ be the $i$ th row of the matrix $\mathbf{X}_{1}=\left(\mathbf{x}_{11}, \ldots, \mathbf{x}_{1 n}\right)^{\top}$. Similarly, let $\mathbf{X}_{2}=\left(\mathbf{x}_{21}, \ldots, \mathbf{x}_{2 n}\right)^{\top}$. The linear model $\sqrt{4.1}$ can be written as

$$
\mathbf{y}=\alpha \mathbf{1}+\mathbf{X}_{1} \boldsymbol{\beta}_{1}+\mathbf{X}_{2} \boldsymbol{\beta}_{2}+\boldsymbol{\epsilon}
$$

with the error vector $\boldsymbol{\epsilon}=\left(\epsilon_{1}, \ldots, \epsilon_{n}\right)^{\top}$ and $\mathbf{1}=(1, \ldots, 1)^{\top}$.

Following the same idea in Section 3, we develop a new test for the hypothesis testing (4.2). For an integer $1 \leq k_{2}<\min \left\{n-p_{1}, p_{2}\right\}$, let $\mathbf{P}_{k_{2}} \in \mathbb{R}^{p_{2} \times k_{2}}$ be a matrix with i.i.d. $\mathcal{N}(0,1)$ entries, drawn independently of the data. We define the following projection matrices:

$$
\begin{aligned}
& \mathbf{P}_{1}=\frac{1}{n} \mathbf{1} \mathbf{1}^{\top} \\
& \mathbf{P}_{\mathbf{X}_{1}}=\left(\mathbf{I}-\mathbf{P}_{1}\right) \mathbf{X}_{1}\left(\mathbf{X}_{1}^{\top}\left(\mathbf{I}-\mathbf{P}_{1}\right) \mathbf{X}_{1}\right)^{-1} \mathbf{X}_{1}^{T}\left(\mathbf{I}-\mathbf{P}_{1}\right) \\
& \mathbf{H}_{k_{2}}=\left(\mathbf{I}-\mathbf{P}_{1}\right) \mathbf{W}\left(\mathbf{W}^{\top}\left(\mathbf{I}-\mathbf{P}_{1}\right) \mathbf{W}\right)^{-1} \mathbf{W}^{\top}\left(\mathbf{I}-\mathbf{P}_{1}\right)
\end{aligned}
$$


where $\mathbf{W}=\left(\mathbf{X}_{1}, \mathbf{X}_{2} \mathbf{P}_{k_{2}}\right)$. Note that the matrix $\mathbf{W}^{\top}\left(\mathbf{I}-\mathbf{P}_{1}\right) \mathbf{W}$ is of full rank with probability 1 when $\mathbf{P}_{k_{2}}$ has i.i.d. $\mathcal{N}(0,1)$ entries and $k_{2}$ is appropriately selected. This ensures the projection matrix $\mathbf{H}_{k_{2}}$ is well-defined, even when $p_{2}>n$. We propose a new test statistic

$$
T_{n, p_{2}}=\frac{\mathbf{y}^{\top}\left(\mathbf{H}_{k_{2}}-\mathbf{P}_{\mathbf{X}_{1}}\right) \mathbf{y} / k_{2}}{\mathbf{y}^{\top}\left(\mathbf{I}-\mathbf{P}_{1}-\mathbf{H}_{k_{2}}\right) \mathbf{y} /\left(n-1-p_{1}-k_{2}\right)}
$$

From the definition, the numerator of $T_{n, p_{2}}$ presents the part of $\mathbf{y}$ that can only be explained by $\mathbf{X}_{2} \mathbf{P}_{k_{2}}$, while the denominator of $T_{n, p_{2}}$ estimates the variance of the error term.

\subsection{Asymptotic Null Distribution}

To study the asymptotic null distribution and the power of the proposed test, we make the following assumptions.

Assumption S1. $\boldsymbol{x}_{i}=\left(\boldsymbol{x}_{1 i}^{\top}, \boldsymbol{x}_{2 i}^{\top}\right)^{\top}=\boldsymbol{\mu}+\boldsymbol{\Gamma} \boldsymbol{z}_{i}$, where $\boldsymbol{x}_{1 i} \in \mathbb{R}^{p_{1}}$ and $\boldsymbol{x}_{2 i} \in \mathbb{R}^{p_{2}}$ are covariates, $\boldsymbol{\mu}$ is a p-dimensional mean vector, $\boldsymbol{\Gamma}$ is a $p \times m$ matrix with $m \geq p$, and $\boldsymbol{z}_{i}$ is an m-variate random vector with $E\left(\boldsymbol{z}_{i}\right)=\boldsymbol{O}, \operatorname{Var}\left(\boldsymbol{z}_{i}\right)=\boldsymbol{I}_{m}$, and $\operatorname{Var}\left(\frac{\boldsymbol{z}_{i}^{\top} \boldsymbol{z}_{i}}{m}\right)=O\left(m^{-1}\right)$. For any nonnegative integers $q_{1}, \ldots, q_{m}$, with $\sum_{j=1}^{m} q_{j}=4$, the mixed moments $E\left(\Pi_{j=1}^{m} z_{i j}^{q_{j}}\right)$ are bounded, and equal 0 when at least one of the $q_{j}$ is odd.

Assumption S2. $\mu_{4}=E\left(\epsilon_{1}^{4}\right)<\infty$.

Assumption S3. $p=p_{1}+p_{2} \gg n, p_{2} \gg p_{1}$, and there exist constants $\rho_{1}, \rho_{2} \in(0,1)$, with $\rho_{1}+\rho_{2}<1$, such that $\frac{p_{1}}{n} \rightarrow \rho_{1}$ and $\frac{k_{2}}{n} \rightarrow \rho_{2}$.

Since $T_{n, p_{2}}$ is invariant to the location shift of $\mathbf{y}, \mathbf{X}_{1}$ and $\mathbf{X}_{2}$, we assume $\alpha=0$ and $\boldsymbol{\mu}=\mathbf{0}$ in the following. The dimensions of the covariates are assumed to satisfy $p_{2} \gg p_{1}$, so $\mathbf{X}_{2}$ 
is the high-dimensional component. In addition, $p_{1}$ is assumed to be less than but can be comparable with $n$. The projection dimension $k_{2}$ needs to be asymptotically proportional to $n$ and the choice of $\rho_{2}$ will be discussed below.

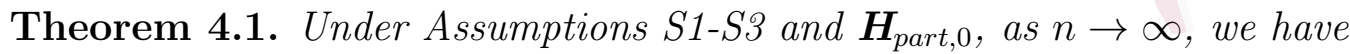

$$
\frac{T_{n, p_{2}}-1}{\sqrt{2\left(1-\rho_{1}\right) / n \rho_{2}\left(1-\rho_{1}-\rho_{2}\right)}} \stackrel{\mathcal{D}}{\longrightarrow} \mathcal{N}(0,1) \text {. }
$$

The asymptotic normality of the standardized test statistic provides the testing procedure. Given a $\alpha$-level of significance, $\mathbf{H}_{\text {part }, 0}$ is rejected when

$$
\frac{T_{n, p_{2}}-1}{\sqrt{2\left(1-\rho_{1}\right) / n \rho_{2}\left(1-\rho_{1}-\rho_{2}\right)}}>z_{\alpha}
$$

where $z_{\alpha}$ is the upper $\alpha$-quantile of $\mathcal{N}(0,1)$.

\subsection{Asymptotic Power Function}

We are now in a position to study the asymptotic power of the test. We first divide $\boldsymbol{\Gamma}=$ $\left(\boldsymbol{\Gamma}_{1}^{\top}, \boldsymbol{\Gamma}_{2}^{\top}\right)^{\top}$ with $\boldsymbol{\Gamma}_{1} \in \mathbb{R}^{p_{1} \times m}$ and $\boldsymbol{\Gamma}_{2} \in \mathbb{R}^{p_{2} \times m}$. Define $\boldsymbol{\Sigma}_{11}=\boldsymbol{\Gamma}_{1} \boldsymbol{\Gamma}_{1}^{\top}, \boldsymbol{\Sigma}_{22}=\boldsymbol{\Gamma}_{2} \boldsymbol{\Gamma}_{2}^{\top}, \boldsymbol{\Sigma}_{12}=\boldsymbol{\Gamma}_{1} \boldsymbol{\Gamma}_{2}^{\top}$ and $\boldsymbol{\Sigma}_{21}=\boldsymbol{\Gamma}_{2} \boldsymbol{\Gamma}_{1}^{\top}$. Following the same idea in Section 3, we give additional assumptions to facilitate our analysis.

Assumption S4. $\boldsymbol{\beta}_{2}^{\top} \boldsymbol{\Sigma}_{22} \boldsymbol{\beta}_{2}=o(1)$, and $\boldsymbol{\beta}_{2}^{\top} \boldsymbol{\Sigma}_{21} \boldsymbol{\Sigma}_{11}^{-1} \boldsymbol{\Sigma}_{12} \boldsymbol{\beta}_{2}=o(1)$.

Assumption S5. The $m$-variate random vector $\boldsymbol{z}_{i}=\left(z_{i 1}, \ldots, z_{i m}\right)^{\top}$ has a Lebesgue density $f_{\boldsymbol{z}}$ and satisfy $E\left(\boldsymbol{z}_{i}\right)=\boldsymbol{O}$ and $\operatorname{Var}\left(\boldsymbol{z}_{i}\right)=\boldsymbol{I}_{m}$. For $j=1, \ldots, m$, the components $z_{i j}$ are 
assumed to be independent, satisfy $E\left(z_{i j}^{20}\right) \leq C$ for a constant $C$, and have the marginal density bounded by a constant $D \geq 1$

Define $\mathbf{V}=\operatorname{diag}\left(\mathbf{I}_{p_{1}}, \mathbf{P}_{k_{2}}\right)$ and $\boldsymbol{\gamma}=\left(\mathbf{V}^{\top} \boldsymbol{\Sigma} \mathbf{V}\right)^{-1} \mathbf{V}^{\top} \boldsymbol{\Sigma} \boldsymbol{\beta}$. We write the p-dimensjonal vector $\mathbf{V} \boldsymbol{\gamma}=\left(\boldsymbol{\xi}_{1}^{\top}, \boldsymbol{\xi}_{2}^{\top}\right)^{\top}$ with $\boldsymbol{\xi}_{1} \in \mathbb{R}^{p_{1}}$ and $\boldsymbol{\xi}_{2} \in \mathbb{R}^{p_{2}}$. Let $\tau_{k}^{2}=\sigma^{2}+\boldsymbol{\beta}^{\top} \boldsymbol{\Sigma} \boldsymbol{\beta}-\boldsymbol{\gamma}^{\top} \mathbf{V}^{\top} \boldsymbol{\Sigma} \mathbf{V} \boldsymbol{\gamma}$. We derive the asymptotic power function of the proposed test.

Theorem 4.2. Under Assumptions S1 S5, we have

$$
\Psi_{n, p_{2}}^{R P}\left(\boldsymbol{\beta}_{2} ; \boldsymbol{P}_{k_{2}}\right)-\Phi\left(-z_{\alpha}+\sqrt{\frac{n\left(1-\rho_{1}-\rho_{2}\right)\left(1-\rho_{1}\right)}{2 \rho_{2}}} \frac{\boldsymbol{\xi}_{2}^{\top}\left(\boldsymbol{\Sigma}_{22}-\boldsymbol{\Sigma}_{21} \boldsymbol{\Sigma}_{11}^{-1} \boldsymbol{\Sigma}_{12}\right) \boldsymbol{\xi}_{2}}{\tau_{k}^{2}}\right) \rightarrow 0
$$

where $\Phi(\cdot)$ is its cumulative distribution function of the standard normal distribution, and $z_{\alpha}$ is the upper $\alpha$-quantile of $\Phi$.

Note that no extra assumption is made for $\boldsymbol{\Sigma}$. From the expression of the asymptotic power function, we can see that the product $\boldsymbol{\xi}_{2}^{\top}\left(\boldsymbol{\Sigma}_{22}-\boldsymbol{\Sigma}_{21} \boldsymbol{\Sigma}_{11}^{-1} \boldsymbol{\Sigma}_{12}\right) \boldsymbol{\xi}_{2}$ is preferred to be larger, which is dependent on $\mathbf{P}_{k_{2}}$ and is upper bounded by $\boldsymbol{\beta}_{2}^{\top}\left(\boldsymbol{\Sigma}_{22}-\boldsymbol{\Sigma}_{21} \boldsymbol{\Sigma}_{11}^{-1} \boldsymbol{\Sigma}_{12}\right) \boldsymbol{\beta}_{2}$. We give a sufficient condition such that the upper bound can be reached.

Assumption S6. There exist an integer $s_{2}<k_{2}$ and a real number $\gamma_{2}>0$, such that $\left\|\boldsymbol{\beta}_{2}\right\|_{2}^{2} \sum_{i=s_{2}+1}^{p_{2}} d_{i}=o\left(p_{2} n^{-0.5-\gamma_{2}}\right)$, where $d_{i}$ are the eigenvalues of $\boldsymbol{\Sigma}_{22}$ satisfying $d_{1} \geq d_{2} \geq$ $\cdots \geq d_{p_{2}} \geq 0$

This assumption ensures Lemma 3.2 is valid for $\boldsymbol{\beta}_{2}$ and $\boldsymbol{\Sigma}_{22}$, leading to a negligible distance between the vector $\boldsymbol{\Gamma}^{\top} \boldsymbol{\beta}$ and the space generated by $\boldsymbol{\Gamma}^{\top} \mathbf{V}$. In this case, we denote the power function of the proposed random-projection-based test $T_{n, p_{2}}$ as $\Psi_{n, p_{2}}^{R P}\left(\boldsymbol{\beta}_{2}\right)$. 
Corollary 4.1. Under Assumptions $S 1 \sim S 6$, we have

$$
\Psi_{n, p_{2}}^{R P}\left(\boldsymbol{\beta}_{2}\right)-\Phi\left(-z_{\alpha}+\sqrt{\frac{n\left(1-\rho_{1}-\rho_{2}\right)\left(1-\rho_{1}\right)}{2 \rho_{2}}} \frac{\boldsymbol{\beta}_{2}^{\top}\left(\boldsymbol{\Sigma}_{22}-\boldsymbol{\Sigma}_{21} \boldsymbol{\Sigma}_{11}^{-1} \boldsymbol{\Sigma}_{12}\right) \boldsymbol{\beta}_{2}}{\sigma^{2}}\right) \rightarrow 0
$$

where $\Phi(\cdot)$ is its cumulative distribution function of the standard normal distribution, and $z_{\alpha}$ is the upper $\alpha$-quantile of $\Phi$.

\section{Numerical Studies}

\subsection{Simulation Studies}

We conduct simulations to evaluate the finite-sample performance of the proposed tests and compare it with the RCV test.

The first simulation study was designed for testing the hypothesis: $\mathbf{H}_{0}: \boldsymbol{\beta}=\mathbf{0}$ versus $\mathbf{H}_{1}$ : $\boldsymbol{\beta} \neq \mathbf{0}$ in the linear regression model

$$
y_{i}=\alpha+\mathbf{x}_{i}^{\top} \boldsymbol{\beta}+\epsilon_{i}
$$

Set $\alpha=2$. Suppose that $\epsilon_{i}$ was generated from $\mathcal{N}(0,1)$ or $t(5) / \sqrt{5 / 3}$, and covariate $\mathbf{x}_{i}$ was generated from $\boldsymbol{\mu}+\boldsymbol{\Sigma}^{1 / 2} \mathbf{z}_{i}$, where $\boldsymbol{\mu}=\left(\mu_{1}, \ldots, \mu_{p}\right)^{\top}$ with $\mu_{i}$ generated from $U(2,3)$ independently, and $\mathbf{z}_{i}=\left(z_{i 1}, \ldots, z_{i p}\right)^{\top}$ was generated as (i) $\mathcal{N}\left(\mathbf{0}, \mathbf{I}_{p}\right)$ or $($ ii $) z_{i j} \stackrel{i . i . d .}{\sim} U(-\sqrt{3}, \sqrt{3})$. The matrix $\Sigma^{1 / 2}$ was generated by $\mathbf{U} \sqrt{\mathbf{D}} \mathbf{U}^{\top}$, where $\mathbf{U}$ was an orthogonal matrix generated from the uniform distribution on the $p \times p$ orthogonal group with the $i$ th column denoted by $\mathbf{u}_{i}$ and $\sqrt{\mathbf{D}}=\operatorname{diag}\left(\sqrt{d_{1}}, \ldots, \sqrt{d_{p}}\right)$. Let $s=\left[n^{0.72}\right]$ and $L=\left[n^{0.8}\right]$. The function $[x]$ takes the 


\subsection{Simulation Studies}

greatest integer less than or equal to the number $x$. To achieve the tail eigenvalue condition, we set $d_{i}=1$, for $i \leq s$, and $d_{i}=(L-s)\left(w_{i} / W\right)$, for $i=s+1, \ldots, p$, where $w_{i}=1 /(i-s)^{4}$ and $W=\sum_{i=s+1}^{p} w_{i}$. Under the alternative hypothesis, regression coefficient $\boldsymbol{\beta}$ was randomly selected from $\operatorname{Span}\left\{\mathbf{u}_{1}, \ldots, \mathbf{u}_{s+M}\right\}$ with $\|\boldsymbol{\beta}\|_{2}^{2}$ taking $0.1,0.2$ and 0.3. Different values of $M$ were considered in the simulations: (i) $M=0$ and (ii) $M=50$. Working under high-dimensional settings, we set $(n, p)=(300,3000),(400,5000),(800,5000)$.

In the simulations, we implemented three types of random-projection-based tests according to the choice of random projection: (i) RP test: applying normal random projection; (ii) multi-RP test: independently generating normal random projection for 10 times and utilizing their mean; (iii) S-RP test: applying sparse random projection defined in (2.7) with $l=400$.

We first report the kernel density estimation of the proposed test statistics under $\mathbf{H}_{0}$ in Figures $1 \mathrm{a}$ and $1 \mathrm{~b}$, showing that the asymptotic null distribution of the proposed tests can be well approximated by the standard normal distribution. Here we chose $\rho=0.4$. The good resemblance to normal distribution confirms the theoretical result in Theorem 3.1 .

Tables 1 and 2 report the empirical powers and sizes of the proposed tests and the $\mathrm{RCV}$ test for $\epsilon$ distributed from $\mathcal{N}(0,1)$ and $\sqrt{3 / 5} t(5)$ based on 2000 simulations. It can be observed that the performances of three proposed tests have negligible differences, which confirms the discussion in Section 2 and suggests the feasible usage of different random projection in the test. The empirical sizes of the proposed tests and the RCV test are close to 0.05 under the null hypothesis. The empirical powers of the proposed tests are decreasing functions of $\rho$, which is consistent with the result in Theorem 3.2. Moreover we can see that 
$5.2 \quad$ Illustrative Examples

the power of the tests are increasing functions of the norm of $\boldsymbol{\beta}$. Compared with the RCV test, the proposed tests are more powerful.

In the second simulation study, we consider the testing problem of the partial regression coefficients in the linear model. The results are given in Figure 1 and Table 1 in the supplementary material. In the third simulation, we conducted numerical comparison with LWT test and LDFF test proposed in Lan, Wang, and Tsai (2014) and Lan et al. (2016), respectively. The simulation results are given in Table 2 in the supplementary material. It demonstrates that our proposed test is applicable in the highly correlated setting and has higher testing power than the competing tests.

\section{$5.2 \quad$ Illustrative Examples}

To illustrate the proposed methods, we consider here two examples.

\subsubsection{Example 1}

We considered a real data set of riboflavin (vitamin B2) production by bacillus subtilis. The data was analyzed by van de Geer et al. (2014) and is available in R package "hdi". The real-valued response variable is the logarithm of the riboflavin production rate and there are $p=4088$ covariates (genes) measuring the logarithm of the expression level of 4088 genes. These measurements are from $n=71$ samples of genetically engineered mutants of bacillus subtilis. We modeled the data with a high-dimensional linear model and obtained the p-values of the proposed tests and the RCV test in Table 3 . It is illustrated that all the tests reject the null hypothesis, indicating a considerable significance of genes expression in 
5.2 Illustrative Examples

predicting riboflavin production rate.

Then we were interested in the significance of partial genes expression. Based on the LASSO, we divided coefficients into two parts $\boldsymbol{\beta}_{1}$ and $\boldsymbol{\beta}_{2}$, where the index of $\boldsymbol{\beta}_{2}$ corresponded to the index of the zero part in $\hat{\boldsymbol{\beta}}^{\text {Lasso }}$. We conducted testing for $\boldsymbol{\beta}_{2}$ and the results are shown in Table 3. These large $p$-values indicate that $\mathbf{H}_{p a r t, 0}$ is accepted and this is consistent with the LASSO result.

\subsubsection{Example 2}

We applied the proposed tests to a more recent data set, which is available for download under accession number GSE50948 in the Gene Expression Omnibus (GEO). In this data set, gene expression profiling using RNA from $n=114$ samples of pretreated patients with HER2-positive (HER2+) tumors was performed. As multiple probes might represent the same gene, measurement for each gene was from the probe with the highest interquartile range. After a natural logarithm transformation, we obtained expression values of 20592 genes. In Prat et al. (2014), the implement of researched-based prediction analysis of microarray 50 (PAM50) subtype predictor to the data reported the predominated subtype within HER2+ disease is HER2-enriched (HER2-E) tumors, which has been found the high expression of HER2-regulated genes (for example, ERBB2, GRB7 and FGFR4) is one of the most important characteristics. To have more understanding of HER2-E subtype, we studied the association between HER2-regulated genes and residual genes, with ERBB2 as an example.

Let the response variable be the gene expression level of ERBB2 and the residual $p=$ 
20591 gene expression levels be the covariates. Suppose that the data follow a linear model, the RCV test and our proposed tests reported a significant relationship by rejecting the null hypothesis, which is shown in Table 4. We moved on to identifying strongly associated genes based on the cooperation of the proposed tests and the LASSO estimation. Let the regression coefficient corresponding to the zeros in the LASSO estimator denote as $\boldsymbol{\beta}_{2}$, the proposed tests for the testing problem of this partial regression coefficient were conducted. The p-values of the global and partial hypothesis testing in the table suggest that genes with nonzero coefficient, ESR1, MAP4K3, TLK1 ect., have significant influences on the gene expression of ERBB2, some of which have already been shown to be important to breast cancer. For example, Prat et al. (2014) indicated the lower expression of luminal-related gene ESR1 is one of important characteristics of HER2-enriched (HER2-E) tumors. GamezPozo et al. (2014) found gene expression of MAP4K3 related to the PI3K pathway, which is strongly associated with response to trastuzumab in HER2 breast cancer. Consequently, the new testing procedures can be helpful in confirming existing knowledge and making new discoveries.

\section{Conclusion}

We have proposed a new testing procedure for the hypothesis testing of high-dimensional linear regression, which involves applying random projection and then working with the classical F-test. The usage of random projection contributes to the feasible replacement of high-dimensional covariates with its projected version. Our test is simple both in form and computation. In addition, we do not assume any explicit relationship between $n$ and $p$, which 
indicates our test accommodates extremely high-dimensional settings. The asymptotic null distribution and power function are derived when $(n, p) \rightarrow \infty$. To show the advantage of the new test, we conduct comparison with another powerful high-dimensional test proposed by Cui, Guo, and Zhong (2018). We find a sufficient condition which ensures our test outperforms the competing test. Our discussion gives concrete suggestion for the choice of $\rho$ in different situations. Extending to the testing problem of the partial linear regression coefficient, we propose another test based on the same idea and derive its asymptotic distribution. Numerical simulations and applications to real data illustrate the finite-sample performances of the proposed tests and demonstrate the feasible usage of different random projection.

\section{Supplementary Materials}

The supplementary materials contain the proofs of lemmas and theorems, and additional numerical results.

\section{Acknowledgements}

The authors are grateful to the Editor, Associate Editor, and two referees for their valuable comments and suggestions that greatly improved the paper. This work is supported in part by the Research Grant Council of Hong Kong (15301218, 15303319), the National Natural Science Foundation of China (No. 11771366), the NSF grant DMS-1916199, and The Hong Kong Polytechnic University (P0030124, P0034285). 


\section{References}

Bai, Z. and Saranadasa, H. (1996). Effect of high dimension: by an example of a two sample problem. Statistica Sinica 6, 311-329.

Bühlmann, P. (2013). Statistical significance in high-dimensional linear models. Bernoulli 19, 1212-1242.

Cui, H., Guo, W. and Zhong, W. (2018). Test for high-dimensional regression coefficients using refitted cross-validation variance estimation. The Annals of Statistics 46, 958-988.

Chen, S. X., Zhang, L. X. and Zhong, P. S. (2010). Tests for high-dimensional covariance matrices. Journal of the American Statistical Association 105, 810-819.

Diaconis, P. and Freedman, D. (1984). Asymptotics of graphical projection pursuit. The Annals of Statistics 12, 793-815.

Fan, J., Guo, S. and Hao, N. (2012). Variance estimation using refitted cross-validation in ultrahigh dimensional regression. Journal of the Royal Statistical Society, Series B $\mathbf{7 4}$, $37-65$.

Gamez-Pozo, A., Carrión, R. M. P., Manso, L., Crespo, C., Mendiola, C., Lopez- Vacas, R., Berges-Soria, J., López, I. Á, Margeli, M., Calero, J. L. B. et al. (2014). The Long-HER study: clinical and molecular analysis of patients with HER2+ advanced breast cancer who become long-term survivors with trastuzumab-based therapy. PloS one 9, e109611.

Huang, C. and Huo, X. (2017). A Statistically and Numerically Efficient Independence Test based on Random Projections and Distance Covariance. arXiv preprint arXiv: 1701.06054. 
Lehmann, E. L. (1959). Optimum invariant tests. Annals of Mathematical Statistics 30, $881-884$

Lan, W., Ding, Y., Fang, Z. and Fang, K. (2016). Testing covariates in high dimension linear regression with latent factors. Journal of Multivariate Analysis 144, 25-37.

Li, P., Hastie, T. J. and Church, K. W. (2006). Very sparse random projections. In Proceedings of the 12th ACM SIGKDD international conference on Knowledge discovery and data mining, 287-296. ACM.

Liu, M., Shang, Z. and Cheng, G. (2018). Nonparametric testing under random projection. arXiv preprint arXiv:1802.06308.

Lopes, M., Jacob, L. and Wainwright, M. J. (2011). A more powerful two-sample test in high dimensions using random projection. In Advances in Neural Information Processing Systems, 1206-1214.

Lan, W., Wang, H. and Tsai, C.L. (2014). Testing covariates in high-dimensional regression. Annals of the Institute of Statistical Mathematics 66, 279-301.

Prat, A., Bianchini, G., Thomas, M., Belousov, A., Cheang, M. C., Koehler, A., Gómez, P., Semiglazov, V., Eiermann, W., Tjulandin, S. et al. (2014). Research-based PAM50 subtype predictor identifies higher responses and improved survival outcomes in HER2positive breast cancer in the NOAH study. Clinical Cancer Research 20, 511-521.

Searle, S. R. and Gruber, M. H. J. (2017). Linear models. Wiley Series in Probability and Statistics. John Wiley \& Sons, Inc., Hoboken, NJ. 
van de Geer, S., Bühlmann, P., Ritov, Y. and Dezeure, R. (2014). On asymptotically optimal confidence regions and tests for high-dimensional models. The Annals of Statistics $\mathbf{4 2 ,}$ 1166-1202.

van der Vaart, A. W. (1998). Asymptotic statistics. Cambridge Series in Statistical and Probabilistic Mathematics 3. Cambridge University Press, Cambridge.

Wang, S. and Cui, H. (2013). Generalized $F$ test for high dimensional linear regression coefficients. Journal of Multivariate Analysis 117, 134-149.

Zhong, P. S. and Chen, S. X. (2011). Tests for high-dimensional regression coefficients with factorial designs. Journal of the American Statistical Association 106, 260-274.

Changyu Liu, Department of Applied Mathematics, The Hong Kong Polytechnic University, Hong Kong, China

E-mail: cy.u.liu@connect.polyu.hk

Xingqiu Zhao, Department of Applied Mathematics, The Hong Kong Polytechnic University, Hong Kong, China

E-mail: xingqiu.zhao@polyu.edu.hk

Jian Huang, Department of Statistics and Actuarial Science, University of Iowa, Iowa, U.S.A. E-mail: jian-huang@uiowa.edu 


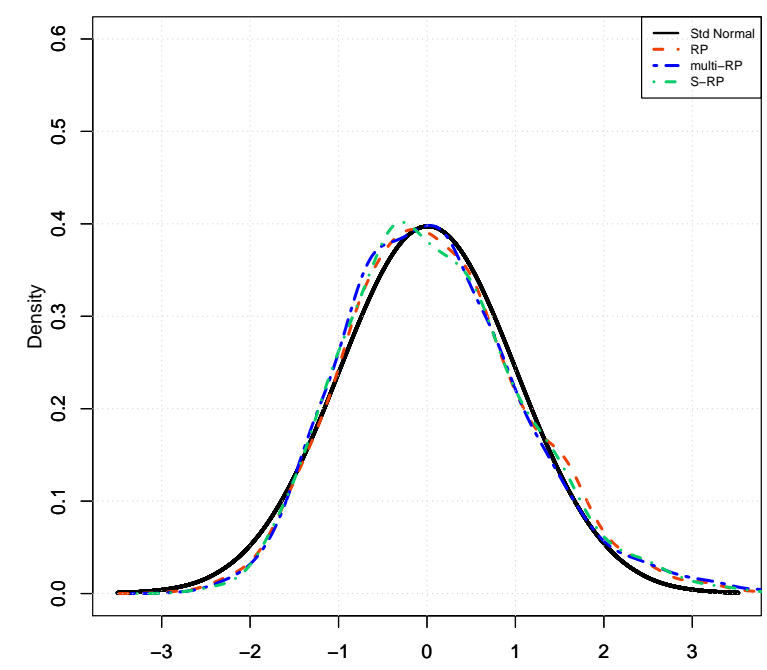

(a) Norm z, norm $\epsilon$ and $(n, p)=(300,3000)$.

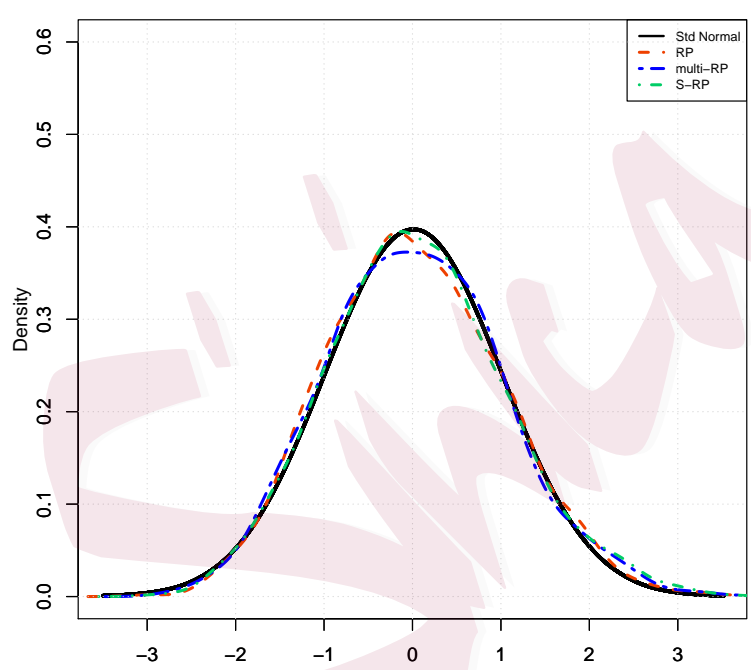

(b) Norm $\mathbf{z}$, norm $\epsilon$ and $(n, p)=(800,5000)$.

Figure 1: The kernel density estimation of RP,multi-RP and S-RP tests under $\mathbf{H}_{0}$. 
Table 1: Empirical Power of RP, multi-RP, S-RP and RCV tests at the significance level 0.05 when $\epsilon \sim \mathcal{N}(0,1)$.

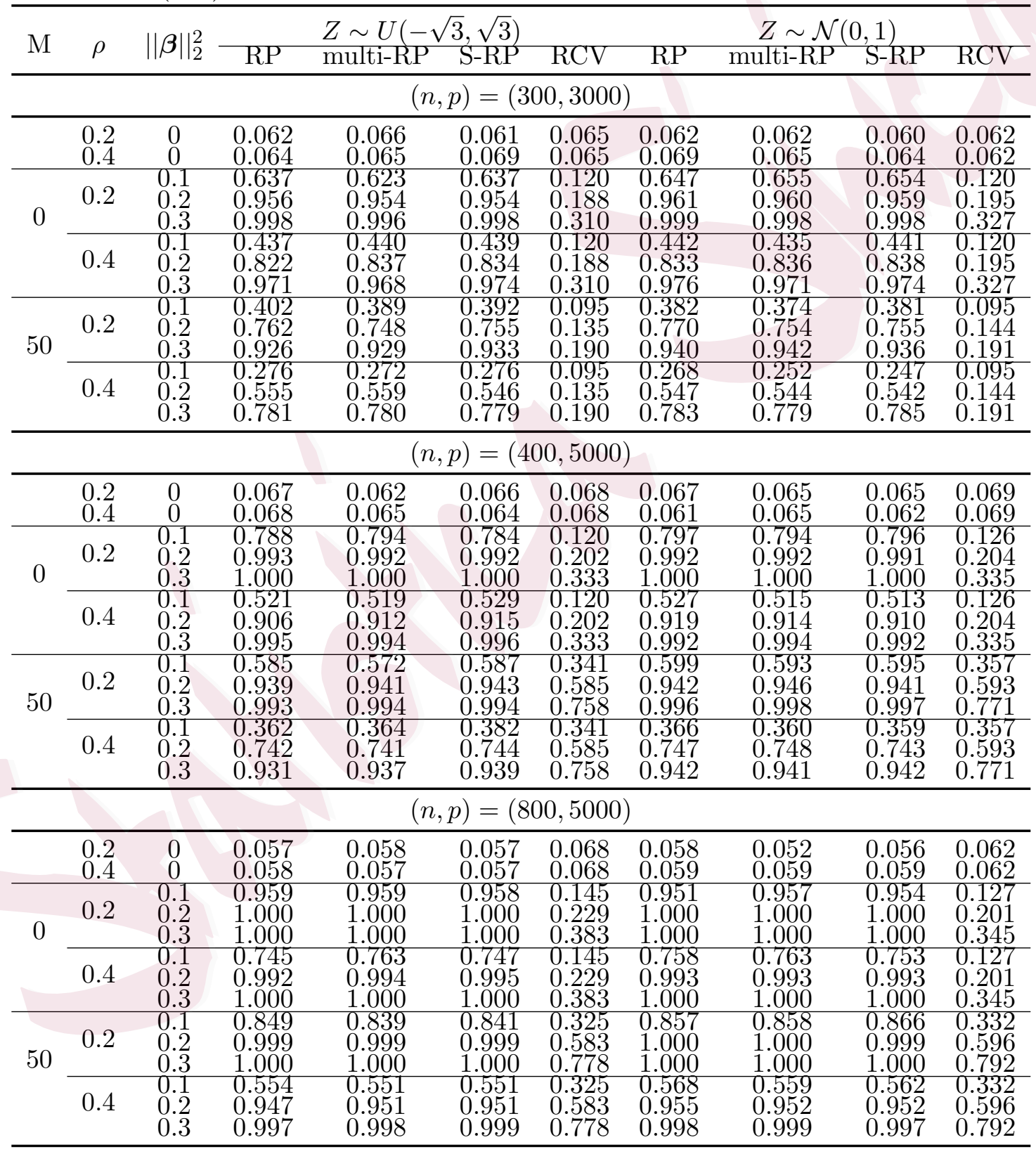


Table 2: Empirical Power of RP, multi-RP, S-RP and RCV tests at the significance level 0.05 when $\epsilon \sim \sqrt{3 / 5} t(5)$.

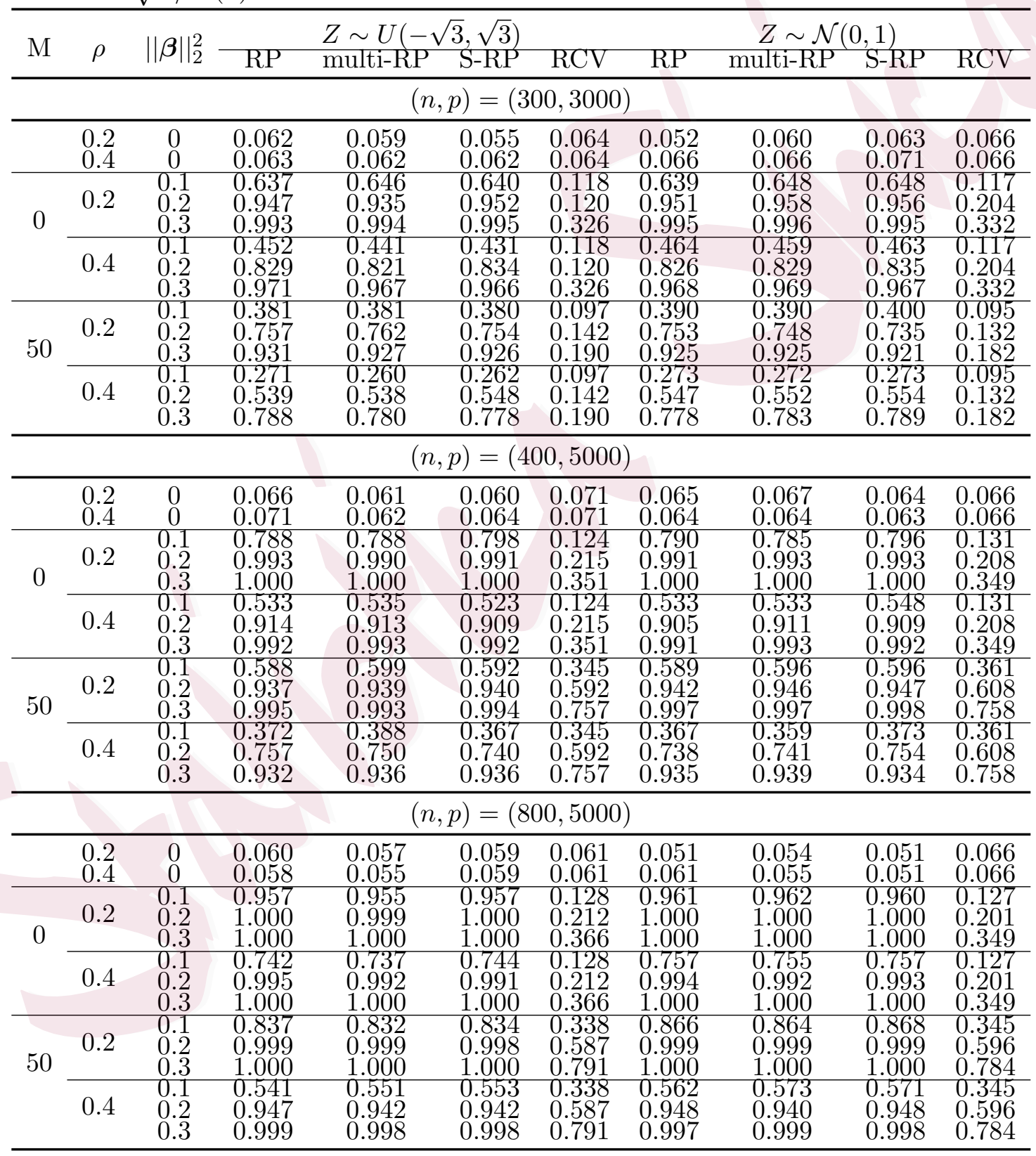


Table 3: The p-values of the proposed tests and the RCV test for example 1.

\begin{tabular}{l|cccc|ccc}
\hline & \multicolumn{3}{|c|}{$\mathbf{H}_{0}: \boldsymbol{\beta}=\mathbf{0}$ vs $\mathbf{H}_{1}: \boldsymbol{\beta} \neq \mathbf{0}$} & \multicolumn{3}{c}{$\mathbf{H}_{\text {part }, 0}: \boldsymbol{\beta}_{2}=\mathbf{0}$ vs $\mathbf{H}_{\text {part }, 1}: \boldsymbol{\beta}_{2} \neq \mathbf{0}$} \\
\hline Tests & RP & multi-RP & S-RP & RCV & RP & multi-RP & S-RP \\
\hline p-value & 0.00 & 0 & 0.00 & 0.00 & 0.73 & 0.61 & 0.65 \\
\hline
\end{tabular}

Table 4: The p-values of the proposed tests and the RCV test for example 2.

\begin{tabular}{l|cccc|ccc}
\hline & \multicolumn{5}{|c|}{$\mathbf{H}_{0}: \boldsymbol{\beta}=\mathbf{0}$ vs $\mathbf{H}_{1}: \boldsymbol{\beta} \neq \mathbf{0}$} & \multicolumn{2}{|c}{$\mathbf{H}_{\text {part }, 0}: \boldsymbol{\beta}_{2}=\mathbf{0}$ vs $\mathbf{H}_{\text {part }, 1}: \boldsymbol{\beta}_{2} \neq \mathbf{0}$} \\
\hline Tests & RP & multi-RP & S-RP & RCV & RP & multi-RP & S-RP \\
\hline p-value & 0.00 & 0.00 & 0.00 & 0.00 & 0.42 & 0.47 & 0.64 \\
\hline
\end{tabular}

